\title{
Symmetric-type dispersion maps in dispersion-managed optical link with mid-span spectral inversion
}

\author{
Jea-Pil Chung ${ }^{1}$, Seong-Real lee ${ }^{2}$ \\ ${ }^{1}$ Department of Electronic Engineering, Gachon University, Republic of Korea \\ ${ }^{2}$ Division of Navigational Information System, Mokpo national Maritime University, Republic of Korea
}

\begin{abstract}
Article Info
ABSTRACT

Article history:

Received Feb 3, 2020

Revised Apr 5, 2020

Accepted Apr 19, 2020

Keywords:

Dispersion map

Dispersion-managed link

Net residual dispersion

Optical phase conjugation

Residual dispersion per span

In long-haul optical communication systems consisting of single-mode fiber spans and fiber amplifiers, such as an erbium-doped fiber amplifier, signal distortion causes performance to deteriorate because of group velocity dispersion and fiber nonlinearity. A combination of dispersion management and optical phase conjugation is an effective technique of compensating for the distortion. In an optical link configured with this combination, the dispersion map mainly affects the compensation for the distorted optical signals. Improvements in system performance have been reported for various types of dispersion maps. In this study, a symmetric type of dispersion map with respect to the midway optical phase conjugator is proposed. The effect of the proposed dispersion maps on the compensation for the distorted 24 channel $\times 40$ Gbps wavelength-division-multiplexed signals was assessed through numerical simulation. It was confirmed that antipodal-type dispersion maps are most appropriate for the compensation, as well as for the flexibility of the link configuration.
\end{abstract}

Copyright $(2020$ Institute of Advanced Engineering and Science. All rights reserved.

\section{Corresponding Author:}

Seong-Real lee,

Division of Navigational Information System,

Mokpo national Maritime University,

Haeyangdaehang-Ro 91, Mokpo, Jeollanam-do, Korea Republic of Korea.

Email: reallee@mmu.ac.kr

\section{INTRODUCTION}

The change in the temporal shape and the spectral change of the optical signal resulting from the chromatic dispersion and Kerr effects of optical fiber disrupt the expansion of the transmission capacity and transmission length of optical communication systems [1-3]. The temporal change gives rise to peaks and valleys in the signal amplitude. Moreover, in ultralong-haul and ultrahigh-data-rate systems, the interaction of the new frequencies induced by an intensity-dependent phase shift, which is produced by the Kerr nonlinear effect, and the fiber chromatic dispersion cause signal distortion [4]. Dispersion management (DM) is very attractive for compensating for the temporal distortion of the optical signal caused by group velocity dispersion (GVD) [1, 5-9]. In a dispersion-managed link, the proper length of the dispersion-compensating fiber (DCF) is used to eliminate or mitigate large positive dispersion accumulated in single-mode fiber (SMF). The DCF is a specially designed fiber with a negative dispersion coefficient; in contrast, the standard SMF has a positive dispersion coefficient of $17 \mathrm{ps} / \mathrm{nm} / \mathrm{km}$. The DCFs are added into the special fiber spans in the lumped DM link, and these are inserted into every fiber span in the in-line DM link. However, for a wavelength-division-multiplexing (WDM) system, it is difficult to compensate for all the channels completely, because the amount of accumulated dispersion of each channel varies with the wavelength [10]. In an ultralong-haul and ultrahigh-data-rate system, although the signal distortion caused by GVD is compensated for by DM, Kerr nonlinear effects cause the system performance to deteriorate, especially at high input power. In this system, a large received power is required for error-free detection [11]; as a result, 
the signal distortion resulting from self-phase modulation (SPM), which is the dominant Kerr effect and is caused by the nonlinear dependence of the refractive index on the signal intensity, is introduced.

Fortunately, optical phase conjugation is a promising solution for compensating for the SPMinduced distortion [4, 12-14]. Mid-span spectral inversion (MSSI), where an optical phase conjugator (OPC) is placed close to the midpoint of the link so that the fiber nonlinearity in the second half of the link mitigates the fiber nonlinearity in the first half $[13,14]$, is an attractive technique of compensation for the distorted signal caused by the Kerr effect. The MSSI technique promises to be transparent to the modulation format [4]. The MSSI technique also makes dispersion compensation possible by placing an OPC that inverts the spectrum and the phase of optical signals distorted by the chromatic dispersion at the middle of a complete transmission link. If phase-conjugated optical signals pass through the same amount of dispersion to the end of the link, phase distortion can be recovered to the initial state, exactly the same as the transmitter output [15]. However, the OPC needs an optimal position, because symmetrical distribution of power and local dispersion with respect to OPC position are required.

Various solutions of combining DM and MSSI have been reported to complement the disadvantages of each technique [16-18]. The mutual interplay between GVD and SPM depends on the sign and amount of residual dispersion [11]. Additionally, the SPM-induced power margin depends on the dispersion map, as well as the DCF's position with respect to the SMF $[11,19]$. Authors also have shown the dependence of compensation degree on the deployment of the SMF and DCF in a dispersion-managed optical link combined with the OPC [20, 21]. In this work, to compensate for the WDM signal distortion caused by the combined effect of GVD and SPM, the various and special dispersion maps are proposed in the dispersion-managed optical link combined with MSSI. All of the dispersion maps proposed are based on the triangular shape in each half transmission link with respect to the midway OPC. Each half transmission link means the whole fiber spans from the first fiber span to the midway OPC (this half is called the "former half section") and from the midway OPC to the last fiber span (this half is the "latter half section"). Four dispersion maps, classified by the symmetric form with respect to the midway OPC, were investigated through numerical simulation to investigate the contribution of compensating for the distorted 960-Gbps WDM signals.

\section{THEORETICAL MODEL AND PERFORMANCE ASSESSMENT METHOD}

The silica optical fibers, including the SMF and DCF, are a lossy, dispersive, and intensitydependent nonlinear medium. Only one expression for the propagation of the optical signal in the silica fiber having these features is the nonlinear Schrödinger equation (NLSE) [22].

$$
\begin{aligned}
& \frac{\partial A_{j}}{\partial z}=-\frac{\alpha}{2} A_{j}-\frac{i}{2} \beta_{2 j} \frac{\partial^{2} A_{j}}{\partial T^{2}}+\frac{1}{6} \beta_{3 j} \frac{\partial^{3} A_{j}}{\partial T^{3}} \\
& +i \gamma_{j}\left|A_{j}\right|^{2} A_{j}+2 i \gamma_{j}\left|A_{k}\right|^{2} A_{j},
\end{aligned}
$$

where $j, k=1,2, \ldots, 24(j \neq k)$ is the WDM channel number, $A_{j}$ represents the slowly varying amplitude of the $j$-th channel's pulse envelope, $z$ is the longitudinal coordinate, i.e., transmission distance, $\alpha$ is the attenuation coefficient of the fiber, $\beta_{2 j}$ is the dispersion parameter, $\beta_{3 j}$ is the dispersion slope, $\gamma_{j}$ is the nonlinear coefficient, and $T=t-z / v_{j}$ is the time measured in a retarded frame at the group velocity $v_{\mathrm{g}}$. The last two terms of Eq. (1) express the effect of nonlinearity, including SPM and cross-phase modulation (XPM). In an optical link consisting of the standard SMF, XPM has less impact on the system performance, because of the high dispersion coefficient of the standard SMF and, hence, its high walk-off [11]. The effect of fourwave mixing (FWM), which is another Kerr effect, on the system performance is neglected because this phenomenon is effectively suppressed by the high dispersion of the standard SMF.

In long-haul transmission using inline amplification, such as an erbium-doped fiber amplifier (EDFA), the additional impairment, the Gordon-Mollenauer (GM) effect, is introduced [4, 23]. The GM effect occurs because of the nonlinear interplay between the signal and the amplified spontaneous emission (ASE) from inline amplifiers. The influence of the accumulated ASE noise of the EDFAs and, consequently, the GM effect on the system performance is excluded in this research to focus on the compensation analysis of the WDM signal distortion resulting from the GVD and SPM effects through the proposed dispersion maps. An easy way to assess the optical pulses that deteriorated because of the GVD and SPM effects is using an eye diagram. The assessment parameters obtained from the eye diagram include the eye-opening penalty (EOP) and quality factor (Q-factor). From the eye diagram, the Q-factor is calculated using the mean values and the standard variations of the mark (" 1 ") pulses and of the space ("0") pulses as follows [24].

$$
\mathrm{Q}-\text { factor }=\frac{I_{1}-I_{0}}{\sigma_{1}+\sigma_{0}}
$$


where $I_{1}$ and $I_{0}$ represent the mean values of " 1 " pulses and " 0 " pulses, respectively, which are obtained at every timing clock period, and $\sigma_{1}$ and $\sigma_{0}$ represent the standard variations of " 1 " pulses and " 0 " pulses, respectively. Generally, 6 is the standard value of the Q-factor for the quasilinear system [24]. Thus, $\mathrm{Q}$-factor $=6$ is used as the reference value in the simulation. That is, if the Q-factor has a value of more than 6 , this case is identified as "good" compensation.

\section{SIMULATION METHOD}

The system configuration, including the dispersion-managed optical link embedded in the midway OPC for simulation, is depicted in Figure 1(a). The dispersion-managed link comprises 50 fiber spans. Thus, $m$ is to be 25, and $n$ is to be 50, as in Figure 1(a). The pre-DC and post-DC in Figure 1(a) mean the predispersion-calibrator and post-dispersion-calibrator, respectively. The dispersion calibrators play the role of the controller of the dispersion amount accumulated in all fiber spans. In other words, they finally control the residual dispersion accumulated across 24 fiber spans of each half section by adjusting the length of the DCF in the first/last fiber span.

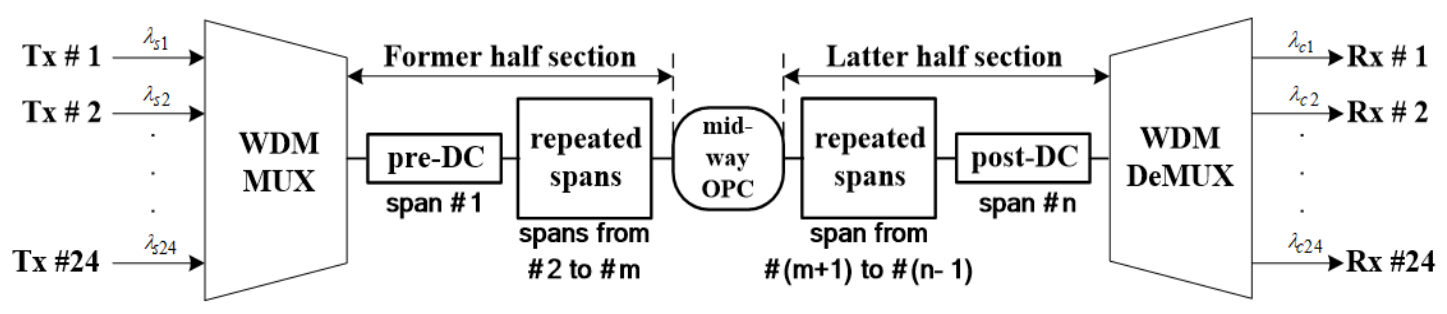

(a) Link configuration

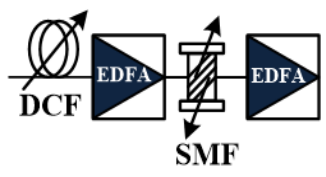

(b) Deployment of fibers in FHS

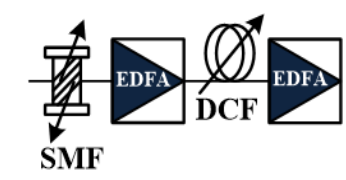

(c) Deployment of fibers in LHS

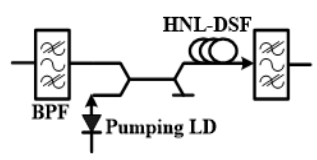

(d) mid-way OPC

Figure 1. The proposed WDM system and dispersion-managed link with the midway OPC for transmitting 960-Gbps

As mentioned earlier, it is important to deploy the SMF and the DCF in each fiber span for good compensation. In every fiber span of the former half section (FHS), the DCF precedes the SMF; however, the reverse order is kept in the latter half section (LHS) [see Figure 1(b) and 1(c)]. This deployment is for a nearperfect symmetry of dispersion distribution with respect to the midway OPC and for the proper position of the pre/post-DC. The parameters of the SMF and the DCF are summarized in Table 1. The length of the DCF depends on the residual dispersion per span (RDPS). The dispersion of one fiber span is completely compensated for when the DCF length is $13.6 \mathrm{~km}$, i.e., RDPS $=0 \mathrm{ps} / \mathrm{nm}$ in this case. However, if the DCF length is less (greater) than $13.6 \mathrm{~km}$, the RDPS is positive (negative). Furthermore, the slope of the straight line in the dispersion map plotted in Figure 2 is determined by the magnitude of the RDPSs over several fiber spans. Two cases of the RDPS (+130/-130 and $+1300 /-1300 \mathrm{ps} / \mathrm{nm})$ are considered for investigating the effect of the slope of the dispersion map on the compensation. The lengths of the DCFs from span \#2 to span \#25 and span \#26 to span \#49 are set to be 12.3 and $14.9 \mathrm{~km}$ (which correspond to RDPSs of +130 and -130 $\mathrm{ps} / \mathrm{nm}$ - represented as "L(low)-slope") or to be 0.6 and $26.6 \mathrm{~km}$ (which correspond to RDPSs of +1300 and $-1300 \mathrm{ps} / \mathrm{nm}$ - represented as "H(high)-slope"). Four types of dispersion maps made from the aforementioned RDPSs are plotted in Figure 2. These are named "S-shape," "S ${ }^{-1}$-shape," "M-shape," and "W-shape". The signs of RDPSs are reversed at span \#13 and span \#38 in each half section for every dispersion map. In Figure 2, the dispersion maps of the $S$ - and $S^{-1}$-shapes are pictorially represented by using the different RDPS value with M- and W-shapes, to distinguish between these shapes. 


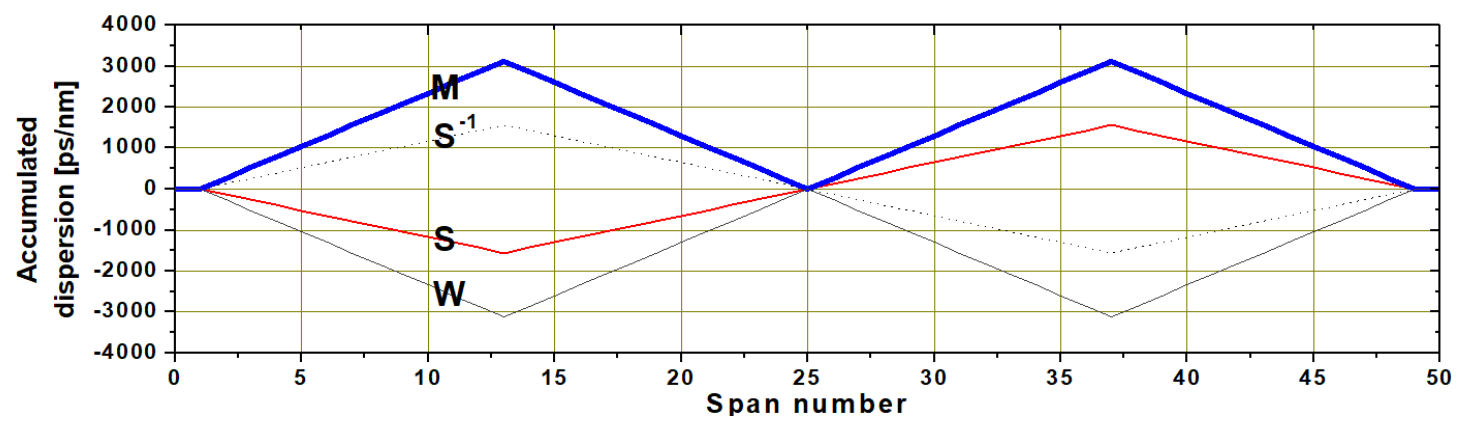

Figure 2. The dispersion maps investigated in this study

Table 1. Fiber parameters

\begin{tabular}{ccc}
\hline Parameters & SMF & DCF \\
\hline Length & $80 \mathrm{~km}$ & Unfixed \\
Attenuation coefficient & $0.2 \mathrm{~dB} / \mathrm{km}$ & $0.6 \mathrm{~dB} / \mathrm{km}$ \\
Dispersion coefficient & $17 \mathrm{ps} / \mathrm{nm} / \mathrm{km}$ & $-100 \mathrm{ps} / \mathrm{nm} / \mathrm{km}$ \\
Dispersion slope & $0.07 \mathrm{ps} / \mathrm{nm} 2 / \mathrm{km}$ & $0.09 \mathrm{ps} / \mathrm{nm} 2 / \mathrm{km}$ \\
Nonlinear coefficient & $1.35 \mathrm{~W}-1 \mathrm{~km}-1$ & $5.06 \mathrm{~W}-1 \mathrm{~km}-1$ \\
\hline
\end{tabular}

In the dispersion-managed link for pseudolinear systems, the optimal net residual dispersion (NRD) should be chosen to be approximately $0 \mathrm{ps} / \mathrm{nm}$ rather than $0 \mathrm{ps} / \mathrm{nm}$ [25]. This result means that the special fiber span has to adjust the optimal NRD, because the total residual dispersion accumulated through 24 fiber spans consisted of each half section becoming $0 \mathrm{ps} / \mathrm{nm}$ in all dispersion maps. The first and last fiber spans were selected as the special fiber spans for this role - the pre-DC and post-DC, respectively. The pre-DC is obtained the following way: the residual dispersion amount is determined by adjusting only the DCF's length of the first fiber span and simultaneously the perfect dispersion elimination in LHS by fixing the DCF's length of the last fiber span to be $13.6 \mathrm{~km}$. The DCF's length of the first fiber span is varied from 13.1 to $14.1 \mathrm{~km}$, correspond to the residual dispersion amounts from 50 to $-50 \mathrm{ps} / \mathrm{nm}$, to find the optimal value. However, the post-DC is obtained the following way: the residual dispersion amount is determined by adjusting only the DCF's length of the last fiber span and simultaneously the perfect dispersion elimination in FHS by fixing the DCF's length of the first fiber span to be $13.6 \mathrm{~km}$. The configuration of the midway OPC is shown in Figure 1(d). Highly nonlinear dispersion-shifted fiber (HNL-DSF) OPC was selected as a phase conjugator for MSSI. The features of HNL-DSF as the nonlinear medium of the OPC are listed in Table 2. The power and wavelength of the pump light for generating the phase-conjugated waves are assumed to be $18.5 \mathrm{dBm}$ and $1549.75 \mathrm{~nm}$, respectively.

Table 2. Features of HNL-DSF

\begin{tabular}{cc}
\hline Parameter & Value \\
\hline Loss & $0.61 \mathrm{~dB} / \mathrm{km}$ \\
Nonlinear coefficient & $20.4 \mathrm{~W}-1 \mathrm{~km}-1$ \\
Length & $0.75 \mathrm{~km}$ \\
Zero dispersion wavelength & $1,550 \mathrm{~nm}$ \\
Dispersion slope & $0.032 \mathrm{ps} / \mathrm{nm} 2 / \mathrm{km}$ \\
\hline
\end{tabular}

Each transmitter (Tx) generates an independent $40-$ Gbps $127\left(=2^{7}-1\right)$ pseudorandom bit sequence. The continuous light waves from each distributed feedback laser diode are injected into the external optical modulator. The center wavelengths of each Tx are selected from 1550 to $1568.4 \mathrm{~nm}$. These are allocated at an interval of $0.8 \mathrm{~nm}(100 \mathrm{GHz})$ according to ITU-T recommendation G.694.1. Finally, return-to-zero (RZ) pulses are generated as the modulated format from the external optical modulator. The output electric field of the RZ format is assumed to be a second-order super-Gaussian pulse as given by

$$
U(0, T)=\exp \left[-\frac{1}{2}\left(\frac{T}{T_{0}}\right)^{2 m}\right]
$$

where $T_{0}$ is the half width at the 1/e-intensity point, and $m$ is the degree of the super-Gaussian pulse. It is assumed that $m$ is 2 and super-Gaussian pulses have a 10-dB extinction ratio, have a duty cycle of 0.5 , and are chirp free. The pulse envelope amplitude $A_{j}(z, T)$ of the $j$-th channel in (1) is given by 


$$
A_{j}(z, T)=\sqrt{P_{0}} \exp \left(-\alpha \frac{z}{2}\right) U(z, T)
$$

where, $P_{0}$ is the peak power.

The RZ signals of 24 channels are multiplexed in an arrayed-waveguide grating multiplexer and then launched into the dispersion-managed link. The distorted optical signals owing to the chromatic dispersion and Kerr nonlinearities of FHS reach the midway OPC. Through the FWM process of the input waves and the pump light, the conjugated waves with wavelengths of $1549.5-1528.5 \mathrm{~nm}$ are generated in in the midway OPC. The ratio of the FWM product power to the input signal power is defined as conversion efficiency [26]. We confirm that the original and conjugated wavelengths belong to the 3 -dB bandwidth (from 1526 to $1574 \mathrm{~nm}$ ).

The 24 conjugated multiplexed channels are propagated through LHS of the link, demultiplexed, and sent into each receiver $(\mathrm{Rx})$ for direct detection. Each Rx consists of an EDFA as a preamplification stage, an optical filter, a photodetector, a pulse-shaping Butterworth filter, and a decision circuit. The noise figure of the EDFA is $5 \mathrm{~dB}$, and the bandwidth of the optical filter is $1 \mathrm{~nm}$ in this study. Modeling of the photodetector is based on the PIN diode. The receiver bandwidth is assumed to be 0.65 times $40 \mathrm{Gbps}$.

\section{SIMULATION RESULTS AND DISCUSSIONS}

Eye diagrams for the pulses received through the proposed DM link with NRD $=10 \mathrm{ps} / \mathrm{nm}$ by preDC are given in Figure 3. These eye diagrams are obtained under the same conditions: RDPS $= \pm 1300 \mathrm{ps} / \mathrm{nm}$ and launch power of $-4 \mathrm{dBm}$. Generally, the eye opening depends on the input power level. In addition to the result as we known, it was confirmed from the results in Figure 3 that the eye opening is also affected by the dispersion map, i.e., the distribution of RDPSs. It was also confirmed that the dispersion map with an antipodal-type configuration, including $\mathrm{S}$-shape and $\mathrm{S}^{-1}$-shape, is advantageous to compensate for the distortion of WDM channels rather than the bilateral-symmetry type, including M-shape and W-shape.

Figure 4 shows Q-factors of the worst channels as a function of NRD for inducing the best NRD value related to DC. The results of Figures 4(a) and (b) were obtained under the condition of a launch power of $-3 \mathrm{dBm}$. Generally, the best NRD values are 10 or $-10 \mathrm{ps} / \mathrm{nm}$; however, these values are affected by the RDPS value, i.e., the performance difference of WDM channels according to the optimal NRD clearly appears in the case of the H-slope rather than the L-slope (the performances at each optimal NRD in the case of the H-slope are very different from those for the L-slope). The Q-factor in the bilateral-symmetry type, i.e., M- and W-shaped dispersion maps, is significantly lower than the distributions of the antipodal type. This feature appears for all launch powers. Therefore, we should mainly focus on the compensation characteristics in S- and $\mathrm{S}^{-1}$-shaped dispersion maps.
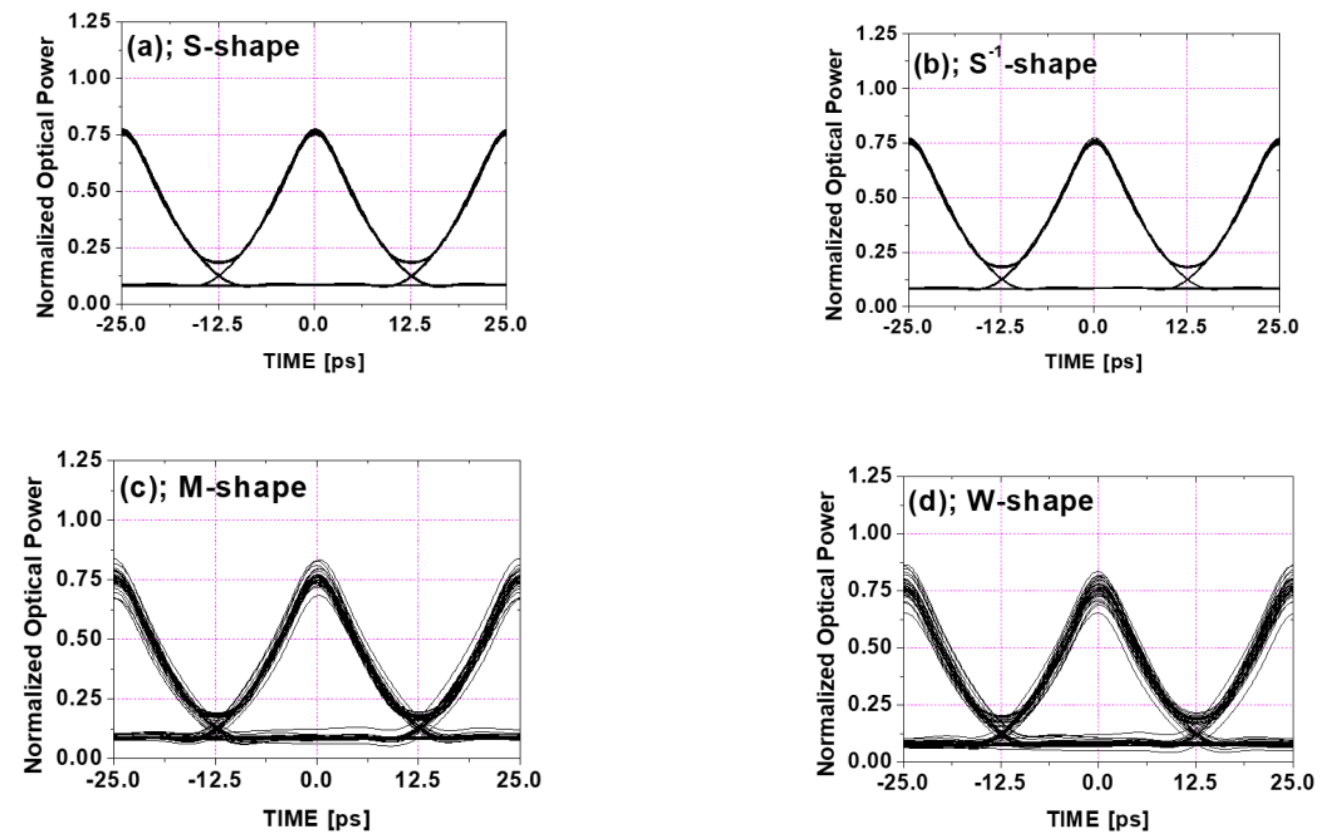

Figure 3. Eye diagrams of the worst channel with $P_{0}=-4 \mathrm{dBm}$ 


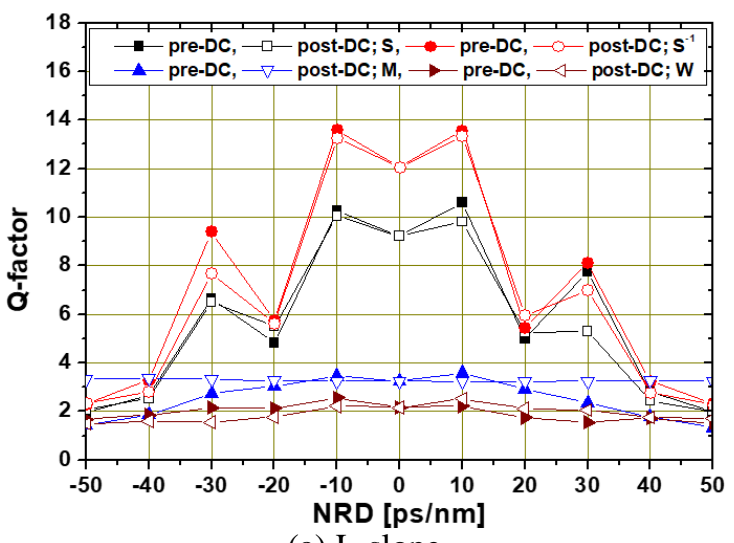

(a) L-slope

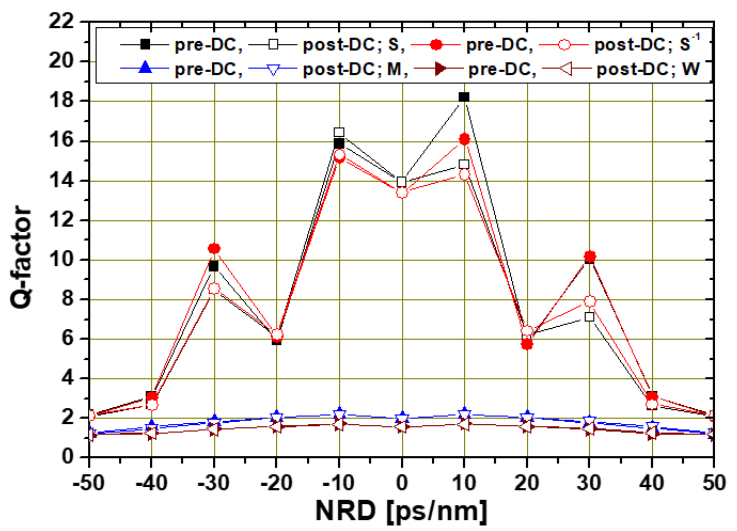

(b) H-slope

Figure 4. NRD versus Q-factor in case of $-3 \mathrm{dBm}$ of the launch power

Figure 5 illustrates Q-factors of the worst channels as a function of the launch power in the case of the fixed NRD with 10 or $-10 \mathrm{ps} / \mathrm{nm}$. The "uniform" legend entry in Figure 5 refers to the DM link consisting of RDPS $=0 \mathrm{ps} / \mathrm{nm}$ for each fiber span, for comparison with the proposed dispersion maps. It is evident that the compensation of the distorted WDM channels in $\mathrm{S}$ - and $\mathrm{S}^{-1}$-shaped dispersion maps is superior to a uniform distribution. The notable feature from Figure 5 is that the compensation through the S-shaped dispersion map more heavily depends on the slope of the dispersion map than the $\mathrm{S}^{-1}$-shaped dispersion map, i.e., the difference of Q-factor between L-slope and H-slope in the S-shape is larger than in the $\mathrm{S}^{-1}$-shape. Therefore, the result in Figure 5 shows that an $\mathrm{S}^{-1}$-shaped dispersion map can provide stable compensation less sensitive to the details in the dispersion-managed link design, while the best compensation is obtained by using the S-shaped dispersion map with the H-slope.
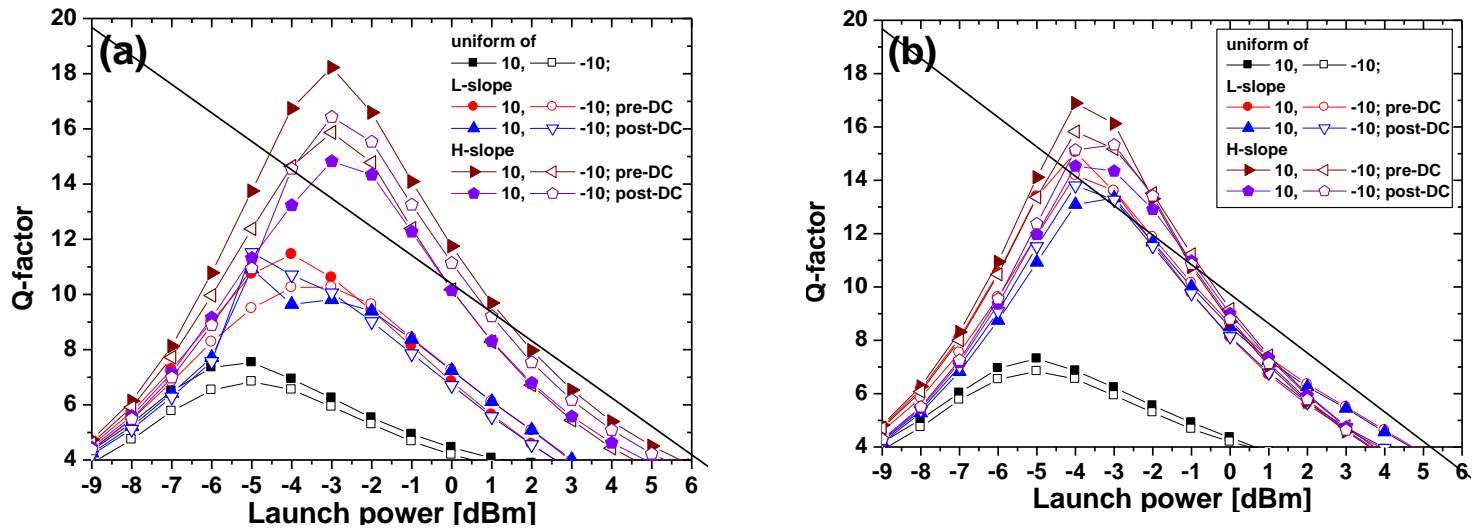

Figure 5. Launch power versus Q-factor of the worst channel: S-shaped dispersion map (a) and $\mathrm{S}^{-1}$-shaped dispersion map (b)

The results in Figure 4 show that the Q-factor of the worst channel is more than 6 at the various NRD values, including the optimal NRD values of 10 and $-10 \mathrm{ps} / \mathrm{nm}$. This result means that it is not necessary to keep the optimal NRD when considering the flexible configuration of the DM link. The NRD value result of a Q-factor above 6 is defined as the effective NRD range to design a flexible DM link. Figure 6 illustrates the worst channel's effective NRD ranges as a function of the launch power, in the three cases of all DM links. The effective NRD range is a contour form - that is, if the values of the $x$ - and $y$-axes within the contour are selected as the launch power and the NRD of the special DM link, respectively, the WDM channels compensated by the DM link with the midway OPC have a Q-factor higher than 6. 


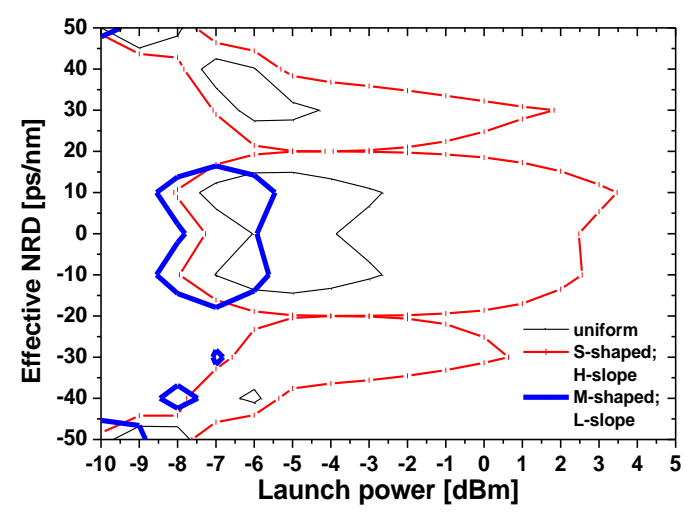

Figure 6. Effective NRD ranges of the worst channel as a function of the launch power

The contour area of the DM link configured with an S-shaped dispersion map of the H-slope is widest among all proposed DM links. This dispersion map is the best way to establish the flexible DM link. The product of NRD and (launch) power is used as the quantitative factor for investigating the effect of each dispersion map on the flexibility. This factor is identical to the area of contour, such as in Figure 6.

The product of NRD and power for the dispersion maps of the antipodal type, as well as the uniform distribution, is plotted in Figure 7. The product of NRD and power of all the proposed antipodal-type dispersion maps is remarkably broader than a uniform distribution, irrelevant of the slope of the dispersion map. In all cases, pre-DC is advantageous for flexible DM link design compared with post-DC. In view of the slope of the dispersion map, the high slope (i.e., $\pm 1300 \mathrm{ps} / \mathrm{nm}$ ) is superior to the low slope. The results of Figure 7 show that it is necessary to compress and expand the distorted WDM channels further to improve the compensation effect in the DM link configured with antipodal-type dispersion maps.

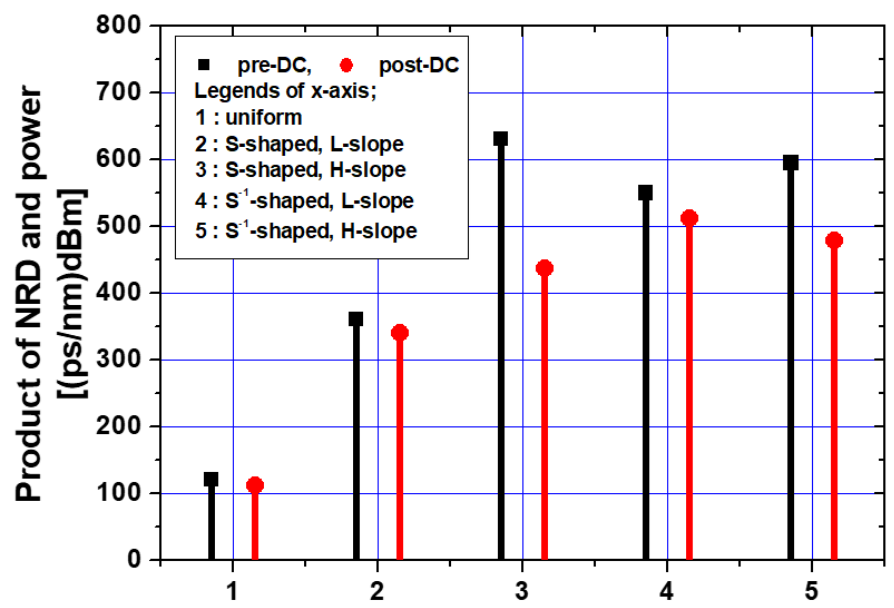

Figure 7. Product of NRD and power

\section{CONCLUSION}

In this study, the effects of dispersion maps of the antipodal and bilateral-symmetry types on the compensation for the distorted 960-Gbps WDM channels in the DM link with midway OPC were investigated. Generally, the antipodal-type dispersion maps are more highly effective for the compensation than the bilateral-symmetry type. In a DM link configured with dispersion maps of the antipodal type, preDC is more advantageous for flexible setup of the DM link than post-DC. It was also confirmed that the flexibility of the link design is more improved as the slope of the dispersion map increases in the dispersion maps of the antipodal type. Consequently, among the proposed dispersion maps, the S-shaped dispersion map with a high slope is the best case for compensation and link flexibility. 


\section{REFERENCES}

[1] M. D. Pelusi, "WDM Signal All-Optical Precompensation of Kerr Nonlinearity in Dispersion-Managed Fibers," IEEE Photonics Technology Letters, vol. 25, no. 1, pp. 71-74, Jan. 2013.

[2] E. B. Desurvire, "Capacity Demand and Technology Challenges for Lightwave Systems in the Next Two Decades," Journal of Lightwave Technology, vol. 24, no. 12, pp. 4697-4710, Dec. 2006.

[3] A. Al-Hayder, et al., "Transmitting Audio via Fiber Optics under Nonlinear Effects and Optimized Tuning Parameters Based on Co-simulation of MATLAB and OptiSystem ${ }^{\mathrm{TM}}$," International Journal of Electrical and Computer Engineering, vol. 10, no. 3, pp. 3253-3260, June 2020.

[4] P. Minzioni, V. Pusino, I. Cristiani, L. Marazzi, M. Martinelli, C. Langrock, M. M. Fejer, and V. Degiorgio., "Optical Phase Conjugation in Phase-Modulated Transmission Systems: Experimental Comparison of Different Nonlinearity-Compensation Methods," Optics Express, vol. 18, no. 17, pp. 18119-18124, Aug. 2010.

[5] T. Almeida, et al., "A Fast Method for Launch Parameter Optimization in Long-Haul Dispersion-Managed Optical Links," Journal of Lightwave Technology, vol. 33, no. 20, pp. 4303-4310, Oct. 2015.

[6] L. Zhu and G. Li, "Folded Digital Backward Propagation for Dispersion-Managed Fiber-optic Transmission," Optics Express, vol. 19, no. 7, pp. 5953-5959, Mar. 2011.

[7] M. Suzuki and N. Edagawa, "Dispersion-Managed High-Capacity Ultra-Long-Haul Transmission," Journal of Lightwave Technology, vol. 21, no. 4, pp. 916-929, May 2003.

[8] Y. Liu, et al., "A Novel Hybrid Photonic Crystal Dispersion Compensating Fiber with Multiple Windows," Optical and Laser Technology, vol. 44, no. 7, pp. 2076-2079, Oct. 2012.

[9] A. Shaban, et al., "A Mitigation of Channel Crosstalk Effect in Dispersion Shifted Fiber Based on Durability of Modulation Technique," International Journal of Electrical and Computer Engineering (IJECE), vol. 10, no. 1, pp. 891-899, Feb. 2020.

[10] A. H. Gnauck, et al., "16×20 Gb/s, 400 km WDM Transmission over NZDSF using a Slope Compensating FiberGrating Module," IEEE Photonics Technology Letters, vol. 12, pp. 437-439, 2000.

[11] M. A. Talukder and M. N. Islam, "Performance of Bi-end Compensation in a Wavelength-division Multiplexed System Considering the Effect of Self Phase Modulation," Optical Engineering, vol. 44, no. 11, pp. 115005-1115005-6, Nov. 2005.

[12] A. Yariv, et al., "Compensation for Channel Dispersion by Nonlinear Optical Phase Conjugation," Optical Letters, vol. 4, pp. 52-54. 1979 .

[13] S. Chandra, et al., "Simultaneous Dispersion and Non-linearity Compensation with Mid-Span Optical Phase Conjugation and Distributed Raman Amplifier for a Sub-carrier Multiplexed Optical Transmission Link," Optics Communications, vol. 279, pp.177-182, Nov. 2007.

[14] M. Morshed, et al., "Mid-Span Spectral Inversion for Coherent Optical OFDM Systems: Fundamental Limits to Performance," Journal of Lightwave Technology, vol. 31, no. 1, pp. 58-66, Jan. 2013.

[15] S. Hur, et al., "10 Gbps WDM Transmission Performance Limits using In-line SOAs and an Optical Phase Conjugator Based on Four-wave Mixing in SOAs As a Mid-Span Spectral Inversion Technique," Optics Express, vol. 14, no. 11, pp. 4589-4600, May 2006.

[16] X. Xiao, et al., "Partial Compensation of Kerr Nonlinearities by Optical Phase Conjugation in Optical Fiber Transmission Systems without Power Symmetry," Optics Communications, vol. 265, no. 1, pp. 326-330, Sep. 2006.

[17] P. Minzioni and A. Schiffini, "Unifying Theory of Compensation Techniques for Intrachannel Nonlinear Effects," Optics Express, vol. 13, no. 21, Oct. 2005.

[18] H. B. Yim and S. R. Lee, "Compensation Characteristics of Optical Signal Distortions in Dispersion-managed Optical Links with Randomly Distributed RDPS," International Journal of Information and Communication Technology, vol. 8, no. 1, pp. 37-49, Jan. 2016.

[19] D. Breuer, et al., "Optimum Dispersion Management for Nonlinear Optical Single-Channel Systems," 1995 Optical Fiber Communications Conference, pp. 196-197, 1995.

[20] H. B. Yim and S. R. Lee, "Compensation for the Distorted WDM Signals through Dispersion-managed Optical Links Combined with Non-midway Ooptical Phase Conjugation," International Journal of Control and Automation, vol. 11, no. 9, pp.1-10, Sep. 2018.

[21] S. R. Lee, "Dispersion-Managed Links Formed of SMFs and DCFs with Irregular Dispersion Coefficients and Span Lengths," Journal of Information Communication Convergence Engineering, vol.16, no.2, pp.67-71, June 2018.

[22] G. P. Agrawal, "Nonlinear Fiber Optics: Its History and Recent Progress," Journal of the Optical Society of America B, vol. 28, no. 12, pp. A1-A10, Dec. 2011.

[23] N. Md. Yusoff, et al., "Performance Improvement for Hybrid L-band Remote Erbium Doped Fiber Amplifier/Raman using Phase Modulator," International Journal of Electrical and Computer Engineering (IJECE), vol. 8, no. 3, pp. 1875-1881, Jun 2018.

[24] I. Ahmed, et al., "Performance of Signal in Dispersion Managed Quasi-Linear High Bit Rate Optical Transmission System," 2013 IEEE 11th Malaysia International Conference on Communications, pp. 528-533. 2013.

[25] R. I. Killey, et al., "Reduction of Intrachannel Nonlinear Distortion in 40-Gb/s-based WDM Transmission over Standard Fiber," IEEE Photonics Technology Letters, vol. 12, no. 12, pp. 1624-1626, Dec. 2000.

[26] K. Inoue, "Four-wave Mixing in an Optical Fiber in the Zero-dispersion Wavelength Region," Journal of Lightwave Technology, vol. 10, no. 11, pp. 1553-1561, Nov. 1992. 


\section{BIOGRAPHIES OF AUTHORS}

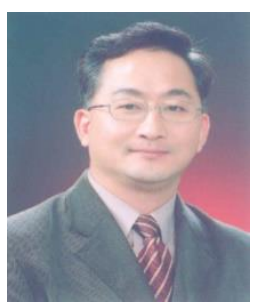

Jae-Pil Chung received his B.S., M.S. from Dankook University, Korea in 1985, 1989, respectively, in alddition to the $\mathrm{Ph}$. D. from Korea Aerospace University, Korea in 2000. He is currently a professor of the Department of Electronic Engineering at Gachon University. His research interests include wireless communication systems, wireless sensor network systems and optical WDM systems.

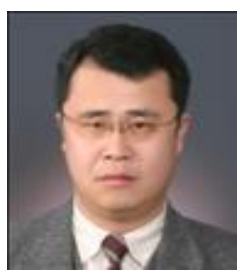

Seong-Real Lee received the B.S., M.S., and Ph.D. degrees in telecommunication and information engineering from Korea Aerospace University, Korea in 1990, 1992, and 2002, respectively. He is currently a professor of Division of Navigational Information System at Mokpo National Maritime University. His research interests include optical WDM systems, optical soliton systems, and optical nonlinear effects. 